\title{
El consumo de tabaco en los hogares mexicanos, 1994-2005
}

\author{
Belén Sáenz de Miera-Juárez, L en Econ, ${ }^{(1)}$ Jorge Alberto Jiménez-Ruiz, M en Econ, (1) \\ Luz Myriam Reynales-Shigematsu, MD, M en C, Dra en C, (') Eduardo Lazcano-Ponce, MD, M en C, Dr en C, (I) \\ Mauricio Hernández-Ávila, MD, PhD.(2)
}

\begin{abstract}
Sáenz de Miera-Juárez B, Jiménez-Ruiz JA, Reynales-Shigematsu LM, Lazcano-Ponce E, Hernández-Ávila M. El consumo de tabaco en los hogares mexicanos, 1994-2005. Salud Publica Mex 2007;49 supl 2:S263-S269.
\end{abstract}

\section{Resumen}

Objetivo. Caracterizar la evolución del consumo de tabaco en los hogares mexicanos entre 1994 y 2005 . Material y métodos. Se estimaron indicadores relevantes (proporciones y medias) a partir de la Encuesta Nacional de Ingresos y Gastos de los Hogares (1994 a 2005). Se presentan intervalos de confianza de los parámetros estimados. Resultados. La prevalencia del consumo de tabaco en hogares se redujo de II.2\% (IC 95\%: I0.4-I2.I) en 1994 a $7.8 \%$ (IC 95\%: 7.3-8.3) en 2005 . El consumo promedio de tabaco se mantuvo estable entre 1994 y 2004. La prevalencia e intensidad del consumo de tabaco entre los hogares de menor ingreso -4.2\% (IC 95\%: 3.4-5.I) y 4.9 cajetillas por mes (IC $95 \%$ : 4.I-5.7) respectivamente- resultaron menores que en los de mayor ingreso -II.2\% (IC 95\%: I0.0-I2.4) y 7.6 cajetillas por mes (IC 95\%: 6.8-8.4) - respectivamente. Por el contrario, el gasto en tabaco como porcentaje del gasto total entre los hogares de menor ingreso-5.5\% (IC 95\%:4.2-6.9)- resultó mayor que entre los de mayor ingreso $-2.5 \%$ (IC 95\%: 2. I-2.8)-. La participación promedio del gasto en cigarros en el gasto total de los hogares fumadores de menor ingreso no presentó diferencias significativas con respecto a la participación del gasto en educación y esparcimiento -8.1\% (IC 95\%: 5.7-10.5)-, vestido y calzado $-4.5 \%$ (IC 95\%: 3.4-5.6) - o cuidados de la salud -4.5\% (IC 95\%:2.6-6.3)-. Conclusiones. A pesar de que la prevalencia del consumo de tabaco en hogares es menor a la de hace una década, el gasto en tabaco ocupa una parte importante del gasto total de los hogares fumadores, en particular, de los que tienen menores ingresos. Dada la escasez de recursos en
Sáenz de Miera-Juárez B, Jiménez-Ruiz JA,

Reynales-Shigematsu LM, Lazcano-Ponce E, Hernández-Ávila M. Household tobacco consumption

in Mexico, 1994-2005.

Salud Publica Mex 2007;49 suppl 2:S263-S269.

\section{Abstract}

Objective. To analyze household tobacco consumption and expenditure in Mexico from 1994 to 2005. Material and Methods. The National Surveys on Household Income and Expenditures conducted in Mexico from 1994 to 2005 were used to estimate relevant statistics (proportions and means). Confidence intervals of the estimated parameters are presented. Results. Smoking household prevalence decreased from II.2\% (IC95\%: I0.4-I2.I) in I 994 to $7.8 \%$ (IC95\%: 7.3-8.3) in 2005. This percentage was lowest in lowincome households $-4.2 \%$ (IC95\%: 3.4-5.I)- and highest in high-income households - I I.2\% (IC95\%: I0.0-12.4). On the contrary, expenditure on tobacco as a percentage of total household expenditures was highest in low-income households -5.5\% (IC95\%: 4.2-6.9)- and lowest in high-income households -2.5\% (IC95\%:2.I-2.8). Conclusion. Household smoking prevalence is lower than a decade before; however, expenditure on tobacco represents a high share of households' total expenditures, particularly, among low-income smoker households. Consequently, tobacco is a burden for low-income smoker households where resources to buy essential goods and services are scarce.

(I) Instituto Nacional de Salud Pública. México.

(2) Secretaría de Salud. México. 
estos últimos, el gasto en tabaco compite con la obtención de satisfactores básicos como alimentos y educación, por lo que representa una carga para ellos.

Palabras clave: consumo de tabaco; gasto en tabaco; tabaquismo; México
Key words: tobacco consumption; expenditure on tobacco; Mexico
$\mathbf{E}^{n}$ n los últimos años, las encuestas de gastos en los hogares se han utilizado cada vez con más frecuencia para analizar el consumo de tabaco en los pa ses de ingresos medios y bajos. ${ }^{1,2}$ Entre otras cosas, esto ha permitido caracterizar la prevalencia e intensidad del consumo de tabaco en hogares en el contexto nacional y por estratos socioeconómicos. En el caso de Indonesia, por ejemplo, se ha documentado una mayor prevalencia en los grupos de menores ingresos, ${ }^{3}$ lo cual coincide con lo encontrado en los últimos años tanto en pa ses de ingresos altos como en pa ses de ingresos medios y bajos. ${ }^{4}$ Además, el análisis de encuestas a hogares ha permitido conocer el papel del gasto en tabaco dentro del presupuesto familiar. En este sentido, diversos estudios muestran que el tabaco es una carga para los usuarios más pobres que destinan una parte de sus recursos, insuficientes para satisfacer sus necesidades básicas, a la adquisición de tabaco. ${ }^{5,6}$

Desde hace varios años, se encuentra disponible en México la Encuesta Nacional de Ingresos y Gastos de los Hogares (ENIGH). Esta encuesta -a cargo del Instituto Nacional de Estad stica, Geograf a e Informática (INEGI)- tiene por objeto ofrecer información detallada sobre el monto, la estructura y la distribución de los ingresos y gasto de los hogares. Debido al elevado grado de desagregación con el que se registran los rubros a los que se destinan los gastos, la ENIGH resulta una fuente de información muy útil para los que se interesan en el estudio de la epidemia del tabaquismo y sus efectos, en particular, en el peso relativo que guarda el gasto en tabaco. Ello reviste especial interés para los hogares de menores ingresos, en los que el costo de oportunidad de los recursos destinados a tabaco es mayor que en el resto de los hogares.

Antes, se hicieron al menos dos estudios sobre el consumo de tabaco en hogares basados en la ENIGH., Ambos coinciden en señalar, dentro de sus respectivos periodos de estudio (1992 a 1998 y 1984 a 2000*), que la prevalencia del consumo de tabaco en los hogares mexicanos se redujo. También muestran que los hogares de menores ingresos destinan una mayor proporción de su

\footnotetext{
* El estudio de Vázquez et al. se actualizó más adelante con los resultados de la ENIGH 2002. ${ }^{9}$
}

ingreso al gasto en tabaco que los hogares de mayores ingresos (aunque los primeros presentan una menor prevalencia del consumo de tabaco).

El objetivo de este trabajo es complementar la información existente sobre el consumo de tabaco en los hogares mexicanos con indicadores precisos de prevalencia, intensidad del consumo y gasto promedio para todas las ediciones de la ENIGH consideradas, dentro de las que se incluyen las más recientes. A diferencia de los estudios previos, en éste no se calcularon prevalencias ajustadas; únicamente se presentan intervalos de confianza para facilitar comparaciones bivariadas, las cuales se complementaron con comparaciones múltiples.

\section{Material y métodos}

Los datos del presente estudio provienen de la ENIGH de 1994 a 2005. El diseño muestral de la ENIGH es polietápico, estratificado y por conglomerados. La unidad última de selección es la vivienda y la unidad de observación es el hogar. Las muestras incluyen viviendas de todas las entidades del pa s y su diseño permite hacer inferencias para toda la población. El número de hogares en cada muestra fue de 12815 en 1994, 13096 en 1996, 10134 en 1998, 10108 en 2000, 17, 167 en 2002, 22595 en 2004 y 23 174 en 2005. El levantamiento siempre se ha llevado a cabo en los meses de agosto a noviembre, excepto el de la ENIGH de 1994, que fue de septiembre a diciembre. Para la captación de la información se utilizan dos instrumentos: 1) un cuestionario básico en el que se registran los datos referentes a las caracter sticas de la vivienda, las caracter sticas de los miembros del hogar, sus ingresos y sus gastos mensuales, trimestrales y semestrales; y 2) un cuadernillo de gastos diarios en el que se registran los gastos diarios en alimentos, bebidas y tabaco. El cuadernillo lo conserva el informante durante la semana de levantamiento, pues se llena a través de entrevistas y de la información que él mismo registra bajo la supervisión del encuestador. ${ }^{10,11}$

A pesar de que las primeras ENIGH se levantaron en 1984, 1989 y 1992, sólo se utilizaron las encuestas de 1994 en adelante para evitar problemas de comparabilidad, ya que a partir de ese año se introdujo 
un cambio importante en la pregunta con la que se recopila la información sobre gasto en tabaco de los hogares. * Las encuestas de 2000 a 2005 que se utilizan están actualizadas con los resultados de la Conciliación Demográfica. ${ }^{\ddagger}$

El gasto en tabaco que se publica en la ENIGH se encuentra ordenado en tres categor as: gasto en cigarrillos, gasto en puros y gasto en tabaco picado. El gasto de más de $99 \%$ de los hogares que informan gasto monetario positivo en tabaco se ubica en la primera categor a. Por ello, para aproximar la prevalencia del consumo de tabaco en hogares, se considera como hogares fumadores a aquellos que notifican gasto monetario positivo en cigarrillos. Para estimar la intensidad del consumo se convirtieron los kilogramos de cigarrillos reportados en la encuesta en cajetillas de 20 cigarrillos. Esto se hizo bajo la suposición de que un kilogramo equivale a 40 cajetillas de 20 cigarrillos cada una. ${ }^{7}$

Los hogares se estratificaron en cinco grupos o quintiles construidos con base en el ingreso corriente total por persona. El ingreso corriente total incluye el ingreso monetario y el no monetario. El ingreso monetario comprende las percepciones en efectivo recibidas por concepto de remuneraciones al trabajo, ingresos netos de negocios propios o cooperativas, ingresos por renta de la propiedad y transferencias. El ingreso no monetario comprende el valor estimado a precios de menudeo de los productos y servicios de autoconsumo y de aquellos recibidos como pago en especie o regalos. También se considera la estimación del alquiler de la vivienda que se hubiera tenido que pagar por la vivienda propia. ${ }^{10,11}$ En el quintil 1 se encuentra agrupado $20 \%$ de los hogares con menor ingreso. En el quintil 5 se encuentra agrupado $20 \%$ de los hogares con mayor ingreso.

\footnotetext{
* En las ENIGH previas a la de 1994, el cuadernillo ten a un apartado especial de tabaco en el que se preguntaba: ¿Qué cantidad de cigarros, puros y tabaco compró en el transcurso de esta semana? Desde la ENIGH de 1994, el tabaco se agrupó con los alimentos y las bebidas, de forma que el cuestionario indica: Anote los Alimentos, Bebidas alcohólicas y no alcohólicas y Tabaco que compraron usted y los miembros del hogar el d a de ayer (la misma instrucción se repite para d a de la semana).

₹ Debido a que al II Conteo de Población y Vivienda 2005 mostró un tamaño de la población inferior en casi 2 millones al dato de las proyecciones que utilizaba el INEGI, se llevó a cabo un ejercicio de conciliación demográfica para reconstruir el comportamiento del tamaño y la estructura de la población para el periodo comprendido entre los años 2000 y 2005. Con base en los resultados de esta conciliación, el INEGI armonizó las bases de datos de las ENIGH de 2000, 2002, 2004 y 2005, para lo cual recalculó el ponderador de las viviendas que conforman las muestras. ${ }^{12}$
}

El procesamiento de las bases de datos se hizo con el paquete estad stico Stata (versión 9.2). Para todas las estimaciones se consideró el diseño muestral de la Encuesta. ${ }^{13}$

\section{Resultados}

La figura 1 muestra la evolución de la prevalencia del consumo de cigarrillos en los hogares mexicanos. Una primera inspección permite ver que el porcentaje de hogares que consumen cigarrillos se redujo al pasar de 11.2\% (IC95\%: 10.4-12.1) en 1994 a $7.8 \%$ (IC95\%: 7.3-8.3) en 2005. En general, si se toma como referencia la prevalencia de 1994, es posible observar que la prevalencia de cualquiera de los años posteriores es menor que la primera. Sin embargo, si se toma como referencia la prevalencia de 2005, se encuentra que las prevalencias de 1998 a 2004 no difieren de ella. ${ }^{*}$ En términos absolutos, la prevalencia del consumo de tabaco del último año corresponde a 2 millones de hogares (IC95\%: 1.9-2.1).

La figura 2 muestra la evolución de la intensidad del consumo de cigarrillos en los hogares fumadores. El consumo promedio de cualquier par de años entre 1994 y 2004 no presenta diferencias. Solamente en 2005 parece registrarse una ca da pronunciada. Si se toma como referencia el consumo promedio de cualquiera de los años previos a 2005, se observa que sólo el consumo del último año es diferente (menor). ${ }^{\ddagger}$

La figura 3 muestra el gasto monetario en cigarrillos como porcentaje del gasto monetario total de los hogares fumadores. En el año 2000 se observa una ligera ca da en este porcentaje con respecto a 1994-de 3.9\% (IC95\%: 3.6-4.2) a 3.1\% (IC95\%: 2.7-3.4)- aunque esta ca da se compensa con el incremento registrado en 2005-cuando alcanzó 3.8\% (IC95\%: 3.4-4.2)-.\$

En el cuadro I se puede ver el comportamiento de la prevalencia y la intensidad del consumo por quinti-

\footnotetext{
* Con la finalidad de complementar las comparaciones de dos variables, se efectuaron comparaciones múltiples ajustadas por el método de Bonferroni. ${ }^{14} \mathrm{Al}$ tomar el año 1994 como referencia, la comparación múltiple mostró que las prevalencia de 1996 a 2005 son diferentes (menores) a la de ese año $(p=0.003, p=0.000, p=$ 0.006 y $p=0.000$, en los últimos tres casos). Al tomar el año 2005 como referencia, sólo las prevalencias de 1994 ( $p=0.000)$ y 1996 ( $p$ $=0.004$ ) resultaron diferentes (mayores) a la de ese año.

₹ Al tomar como referencia cualquier año distinto de 2005, la comparación múltiple mostró que el consumo promedio del último año es diferente (menor) al resto ( $p=0.000$ en todos los casos), mientras que el de los otros años no presentaba diferencias ( $p>0.05$ en todos los casos).

§ Al tomar el año 2000 como referencia, la comparación múltiple mostró que sólo los porcentajes de 1994 y 2005 resultaron diferentes (mayores; $p=0.007$ y $p=0.016$ ). Al tomar cualquiera de los otros años como referencia, no se encontraron más diferencias.
} 


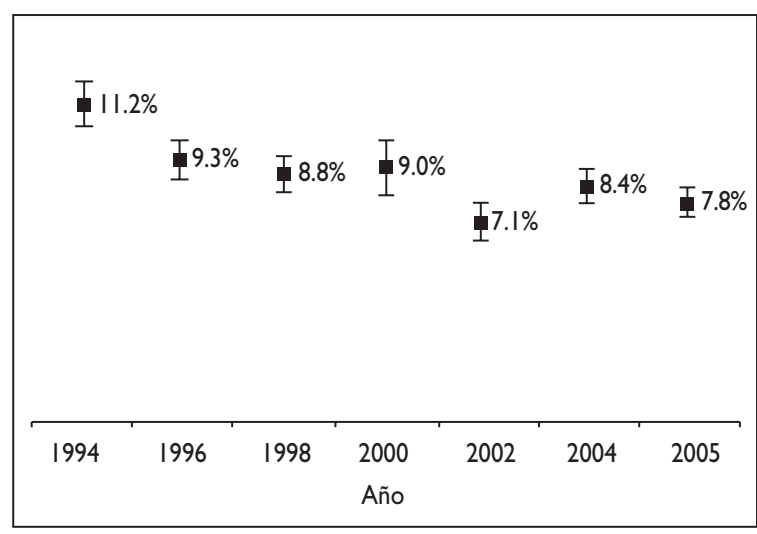

Nota: las líneas verticales representan intervalos de confianza al $95 \%$.

Fuente: elaboración propia con información de la ENIGH.

\section{Figura I. Prevalencia del consumo de cigarrillos en hogares. México, 1994-2005}

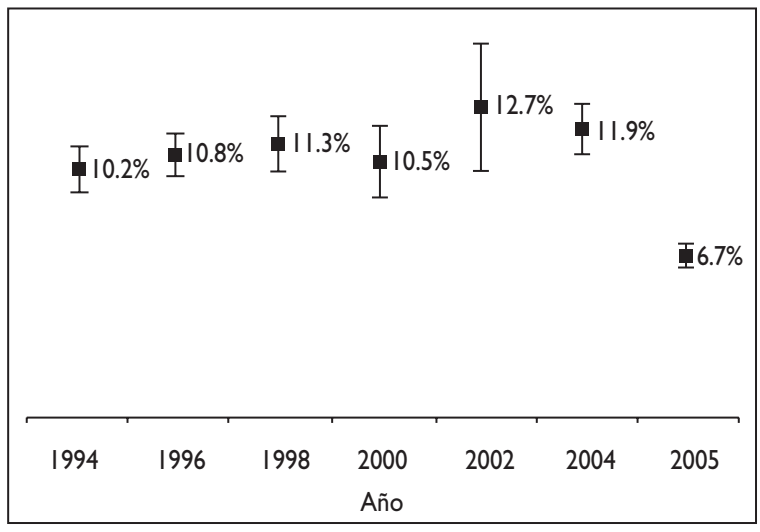

Notas:

Hogar fumador $=$ hogar con gasto positivo en cigarrillos.

Las líneas verticales representan intervalos de confianza al 95\%.

Cajetillas de 20 cigarrillos.

Fuente: elaboración propia con información de la ENIGH.

Figura 2. Consumo promedio mensual de cajetillas DE CIGARRILLOS POR HOGAR FUMADOR. MÉXICO, 1994-2005

les de ingreso para el año 2005. En general, se observa que la prevalencia en los quintiles de mayor ingreso es mayor que la de los quintiles de menor ingreso. Las únicas prevalencias que no presentan diferencias son las de los quintiles 2 y 4 al compararlas con la del tercero

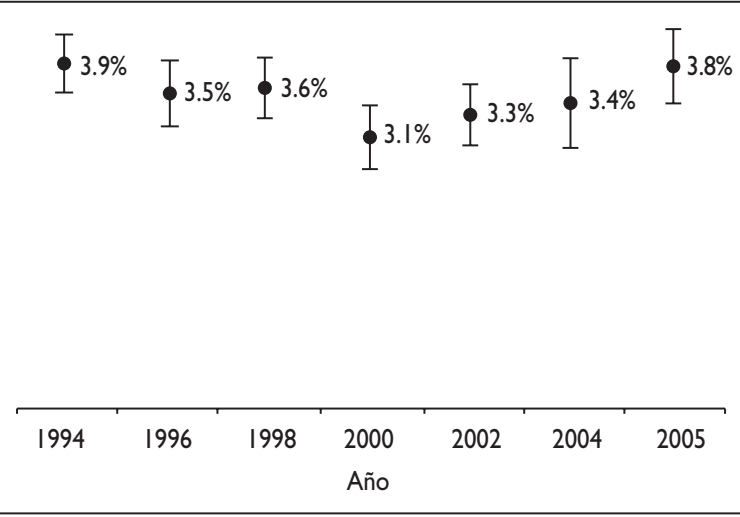

Notas:

Hogar fumador = hogar con gasto positivo en cigarrillos.

Las líneas verticales representan intervalos de confianza al 95\%.

Fuente: elaboración propia con información de la ENIGH.

Figura 3. Gasto monetario en CigarRillos como PORCENTAJE DEL GASTO MONETARIO TOTAL DE LOS HOGARES FUMADORES. MÉXICO, 1994-2005

y las de los quintiles 4 y 5.* En cuanto a la intensidad del consumo, se observa que los hogares fumadores del quintil de menor ingreso consumen en promedio menos cigarrillos que los hogares de los dos quintiles de mayor ingreso. Empero, no se encontraron diferencias entre los consumos promedio de los demás quintiles. También se muestra en el cuadro I el gasto en cigarrillos como porcentaje del gasto total por quintiles para 2005. El porcentaje del quintil 5 resultó menor al de cualquiera de los otros quintiles, pero no se encontraron diferencias entre los porcentajes de los quintiles 1 a 4 .

Finalmente, en la figura 4 se muestra la distribución promedio del gasto monetario de los hogares fumadores en los principales rubros para 2005. La mayor parte del gasto de estos hogares se destina a alimentos $-34.3 \%$ (IC95\%: 33.3-35.3)-. El gasto en educación y esparcimiento $-10.6 \%$ (IC95\%: 9.9-11.4)- y el gasto en vestido y calzado $-5.5 \%$ (IC95\%: 5.1,5.8)- ocupan el segundo y tercer lugar, respectivamente. El gasto en

* Al tomar el quintil 3 como referencia, la comparación múltiple mostró que las prevalencias de los quintiles 2 y 4 no mostraron diferencias ( $p=0.103$ y $p=0.618$ ). De la misma forma, al tomar el quintil 5 como referencia se comprobó que el quintil 4 no presentaba diferencias $(p=0.175)$. 
Cuadro I

Prevalencia del consumo de cigarrillos, intensidad del Consumo de CigarRillos y gasto en CigarRiLlos por QUINTILES DE INGRESO. MÉXICO, 2005

\begin{tabular}{ccccccc}
\multicolumn{7}{c}{ Quintiles de ingreso } \\
\hline Prevalencia (\%) & Q1 & Q2 & Q3 & Q4 & Q5 \\
& 4.2 & 6.3 & 8.0 & 9.3 & 11.2 \\
& {$[3.4-5.1]$} & {$[5.3-7.4]$} & {$[6.9-9.3]$} & {$[8.1-10.7]$} & {$[10.0-12.4]$} \\
\hline
\end{tabular}
Consumo promedio mensual
por hogar fumador*

4.9

[4.I-5.7]

[5.I-7.3]

[5.5-7.8]

[6.2-7.9]

7.6

Gasto en cigarrillos como \%

del gasto monetario total

de los hogares fumadores

4.0

[3.2-4.9]

3.7

4.7

2.5

$[4.2-6.9]$

[3.0-4.5]

[3.4-5.9]

[2.1-2.8]

* Cajetillas de 20 cigarrillos.

Notas:

Hogar fumador $=$ hogar con gasto positivo en cigarrillos.

Entre corchetes se presentan intervalos de confianza al $95 \%$.

Los quintiles están construidos con base en el ingreso corriente total por persona.

El quintil I corresponde a $20 \%$ de los hogares con menor ingreso.

Fuente: elaboración propia con información de la ENIGH.

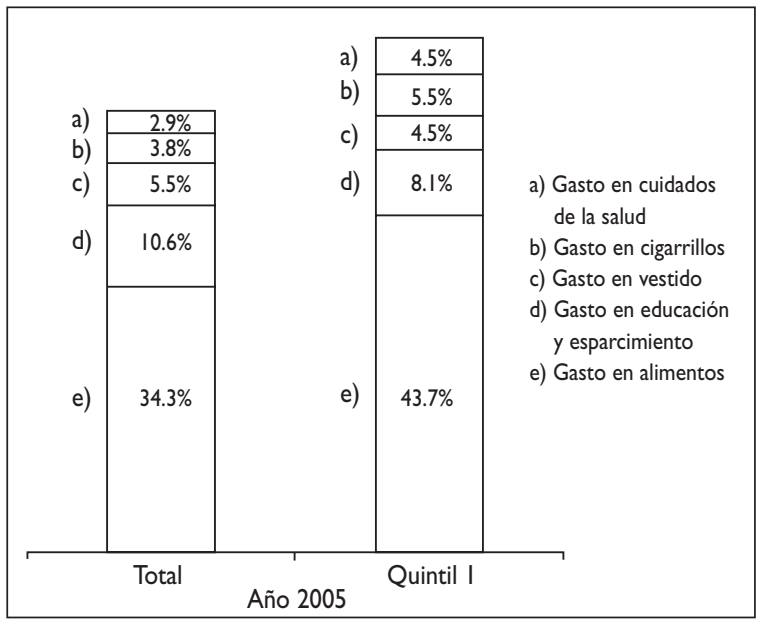

Notas:

Hogar fumador $=$ hogar con gasto positivo en cigarrillos.

El gasto en alimentos no incluye el gasto en bebidas alcohólicas.

Los quintiles están construidos con base en el ingreso corriente total por persona.

El quintil I corresponde a $20 \%$ de los hogares con menor ingreso.

Fuente: elaboración propia con información de la ENIGH.

Figura 4. Gasto promedio de los hogares fumadoRES EN RUBROS SELECCIONADOS COMO PORCENTAJE DE SU gasto monetario total. México, 2005 cigarrillos, con una participación promedio de $3.8 \%$ en el gasto total (IC95\%: 3.4,4.2), se encuentra por debajo de los tres rubros de gasto mencionados, aunque cerca de los últimos dos, y por encima del gasto en cuidados de la salud, el cual tiene una participación promedio de 2.9\% (IC95\%: 2.5-3.3). Si se centra la atención sólo en los hogares fumadores más pobres, resulta posible ver que el gasto en alimentos representa casi la mitad de su gasto total. La participación promedio del gasto en cigarrillos en el gasto total de estos hogares $-5.5 \%$ (IC95\%: 4.2-6.9)-no presenta diferencias significativas con respecto a la participación del gasto en educación y esparcimiento -8.1\% (IC95\%: 5.7-10.5)-, vestido y calzado $-4.5 \%$ (IC95\%: 3.4-5.6)- o cuidados de la salud $-4.5 \%$ (IC95\%: 2.6-6.3)-.

Es importante señalar que lo observado en el cuadro I y la figura 4 para el año 2005 es similar a lo que ocurre al analizar el resto de las ediciones de la ENIGH.

\section{Discusión}

Una de las desventajas de las encuestas, ya sean de hogares o de individuos, es que adolecen de un subreporte del consumo. ${ }^{15}$ Además, de manera habitual, las encuestas a hogares no distinguen a los miembros del hogar 
que consumen tabaco. No obstante, encuestas como la utilizada tienen la ventaja de contar con información muy detallada del ingreso y gasto de los hogares, lo que permite analizar con bastante precisión el consumo por grupos de ingreso, as como estudiar el peso del gasto en tabaco en el gasto total de los hogares. Por otro lado, a pesar de que no se pueden hacer inferencias sobre la prevalencia en individuos a partir de la prevalencia en hogares, es interesante analizar la segunda, ya que los hogares libres de humo de tabaco benefician también a los no fumadores al evitar su exposición al humo de tabaco de segunda mano. ${ }^{3}$

En este trabajo, al igual que en estudios anteriores, ${ }^{4,5}$ se encontró que la prevalencia del consumo de tabaco en los hogares mexicanos se redujo, si bien, dado que la prevalencia del último año no presenta diferencias con respecto a las prevalencias de 1998 a 2004, parece que después de la reducción inicial la prevalencia se estabilizó.

En cuanto a la intensidad del consumo de los hogares fumadores, ya se estudió que entre 1994 y 2004 se mantuvo estable. En consecuencia, es muy probable que la acentuada reducción registrada en 2005 se deba a deficiencias de la información de la encuesta correspondiente. En general, es necesario subrayar que la ENIGH presenta una gran debilidad en lo que se refiere a la captura de la cantidad de cigarrillos, pues la cantidad reconocida por los hogares -normalmente en unidades de cigarrillos o cajetillas- se convierte en gramos, lo que aumenta la posibilidad de incurrir en errores.

A diferencia de los hallazgos en otros pa ses, aunque en consonancia con lo hallado en estudios previos para México, ${ }^{7,8}$ en este trabajo se encontró una mayor prevalencia e intensidad del consumo de tabaco en los hogares de mayor ingreso. En este sentido, es importante mencionar que el número promedio de adultos en los hogares fumadores del quintil 5 es menor que el de los hogares del quintil 1, es decir, la mayor prevalencia e intensidad del consumo en los primeros no se puede atribuir a la presencia de un mayor número de adultos. ${ }^{16}$

Además, se pudo ver que los hogares fumadores con menores ingresos destinan una mayor proporción de su gasto total a la adquisición de tabaco. Un comportamiento similar se ha encontrado en pa ses como Brasil, Chile y Myanmar. ${ }^{17-19}$ Éste es, sin duda, uno de los resultados principales, pues como ya se comentó, los recursos que los hogares pobres gastan en tabaco tienen un mayor costo de oportunidad. Más aún, el tabaco empeora la pobreza entre sus usuarios, entre otras cosas, porque la atención de las enfermedades asociadas con su consumo incrementa el gasto en salud y porque el gasto en tabaco reduce los recursos que podr an destinarse a otros satisfactores básicos como alimentos y educación. 5,6

Para finalizar, es importante señalar la necesidad de complementar los indicadores de consumo presentados en este estudio con otros basados en datos agregados o en encuestas a individuos. ${ }^{20-22}$ De esa manera, se pueden aprovechar las ventajas propias de cada fuente de información para contar con una imagen más clara de la epidemia del tabaquismo en México.

\section{Conclusiones}

Con el objetivo de diseñar una estrategia adecuada para el control del tabaco, en las últimas décadas se fortaleció la vigilancia epidemiológica del tabaquismo tanto en el mundo como en México. El objetivo de este trabajo es colaborar en el bosquejo del panorama epidemiológico del tabaquismo en México y de algunos de sus efectos.

Los resultados muestran que la prevalencia actual del consumo de cigarrillos en hogares es menor a la de hace una década, aunque parece que en los últimos años se estabilizó. La intensidad del consumo muestra un comportamiento constante en el periodo analizado.

Además, se observa que el gasto en cigarrillos ocupa una parte importante del gasto total de los hogares fumadores, en especial, de los que tienen menores ingresos; aunque los hogares del quintil de menor ingreso (1) presentan una menor prevalencia e intensidad del consumo de cigarrillos en comparación con los del quintil de mayor ingreso (5), los primeros destinan una mayor proporción de su gasto total a la compra de cigarrillos. De esta forma, es posible concluir que el tabaco constituye una carga para los usuarios más pobres, pues el gasto en cigarrillos desv a recursos escasos que podr an destinarse a alimentación, salud o educación. Por lo tanto, la búsqueda de mecanismos para el control del tabaco en grupos de bajos ingresos debe ser una prioridad para los que toman decisiones.

\section{Agradecimientos}

El análisis de la información y la redacción de este art culo fueron posibles gracias al apoyo parcial de Fogarty International Center of the National Institutes of Health de los Estados Unidos a través del financiamiento No. RO1-HL-73699. 


\section{Referencias}

I. Onder Z.The Economics of Tobacco in Turkey. New Evidence and Demand Estimates. Washington, DC: Organización Panamericana de la Salud, 2002

2. Nassar H.The Economics of Tobacco in Egypt.A New Analysis of Demand. Washington, DC: Organización Panamericana de la Salud, 2002. 3. Moertiningsih A, Djutaharta T, Hendratno. Cigarette Consumption, Taxation, and Household Income: Indonesia Case Study. Washington, DC Organización Panamericana de la Salud, 2002.

4. Jha P, Chaloupka F. Curbing the epidemic. Governments and the Economics of Tobacco Control,Washington, DC: Banco Mundial, 1999.

5. Efroymson D,Ahmed S. Hungry for Tobacco. An analysis of the economic impact of tobacco on the poor in Bangladesh. 2a edición. Dhaka: PATH Canada, 200I.

6. Efroymson D. FitzGerald S, ed. 5 Tobacco and Poverty. Observations from India and Bangladesh. 2a edición. Dhaka PATH Canada, 2003.

7. Sesma S, Campuzano JC, Carreón VG, Knaul F, López FJ, Hernández M. El comportamiento de la demanda de tabaco en México: 1992-1998. Salud Publica Mex 2002;44 (I):S82-S92.

8.Vázquez LA, Sesma S, Hernández M. El consumo de tabaco en los hogares en México: resultados de la Encuesta de Ingresos y Gasto de los Hogares, 1984-2000. Salud Publica Mex 2002;44 (I):S76-S8I.

9.Vázquez LA,Valdés R, Hernández M. Consumo de tabaco en hogares: Encuesta Nacional de Ingreso Gasto de los Hogares, México, 2002. En: Valdés R, Lazcano EC, Hernández M, ed. Primer informe sobre combate al tabaquismo. México ante el Convenio Marco para el Control del Tabaco. Cuernavaca, México: Instituto Nacional de Salud Pública, 2005: I I5-I23. 10. Instituto Nacional de Estadística, Geografía e Informática. Encuesta Nacional de Ingresos y Gastos de los Hogares. Aguascalientes: INEGI, 2000. (Discos compactos).

I I. Instituto Nacional de Estadística, Geografía e Informática. Bases de datos de la Encuesta Nacional de Ingresos y Gastos de los Hogares para 2000, 2002, 2004 y 2005, armonizadas de acuerdo con la conciliación demográfica. Disponible en: URL: http://www.inegi.gob.mx/est/contenidos/ espanol/ sistemas/enigh/bd/default.asp.
12. Instituto Nacional de Estadística, Geografía e Informática. Comunicado $N^{\circ}$ 209. Disponible en: URL:http://www.inegi.gob.mx/inegi/contenidos/ espanol/prensa/Boletines/Boletin/Comunicados/Especiales/2006/ Septiembre/comunical6.pdf.

13. StataCorp. Survey Data Referente Manual. Release 9. Texas: Stata Press, 2005.

14. Seber GAF. Linear Regression Analysis. Nueva York: John Wiley and Sons, 1977.

I5. Guindon GE, Boisclair D. Tendencias pasadas, presentes y futuras del consumo de tabaco. Washington, DC: Organización Panamericana de la Salud, 2004.

16. Sayginsoy O,Yurekli A, de Beyer O. Cigarette demand, taxation, and the poor. A Case Study of Bulgaria.Washington, DC: Organización Panamericana de la Salud, 2004.

17. Iglesias R.A Economia do controle do tabaco nos países do Mercosul e associados. Brasil. Washington, DC: Organización Panamericana de la Salud, 2006.

18. Debrott D. Economía del control del tabaco en los países del Mercosur y Estados asociados. Chile. Washington, DC: Organización Panamericana de la Salud, 2006.

19. Kyaing NN, Perucic AM, Rahman K. Study on Poverty Alleviation and Tobacco Control in Myanmar. Washington, DC: Organización Panamericana de la Salud, 2004.

20. Instituto Nacional de Estadística, Geografía e Informática. Encuesta Industrial Mensual. Disponible en: URL: http://www.inegi.gob.mx/est/ default.aspx?c $=4398$.

2I. Instituto Nacional de Estadística, Geografía e Informática. Encuesta Nacional de Adicciones 2002. Aguascalientes: INEGI, 2004.

22. Valdés R, Meneses F, Lazcano EC, Hernández MI, Hernández M. Encuesta sobre Tabaquismo en Jóvenes, México 2003. Cuernavaca, México: Instituto Nacional de Salud Pública, 2004. 